\title{
Improving instructional effec
with computer-mediated communication
}

\author{
Som Naidu, John Barrett and Peter Olsen \\ University of Southern Queensland, Australia
}

This study explores the use of asynchronous Computer-Mediated Communication (CMC) in the delivery of instructional content, and points up the interaction among learners, as well as between learners and instructors. The instructional content in the project described was available to learners online as Microsoft Word documents, with email being used for communicating within the student group. Many students, as well as some of the instructors, felt uncomfortable with the flexibility and openness that a CMC environment allowed. However, once familiar.with this process of instruction and interaction, learners were able to work consistently at their own pace, and understand that instructors are interested in every individual learner's opinion and in the collective views of the group. It was evident that a CMC-based instructional delivery system, when carefully planned, has the potential to facilitate that outcome, and to improve instructional effectiveness.

\section{Introduction}

A critical question that often faces educational technologists is how to deliver excellence in teaching and subject-matter content to learners. A corollary to this question is how and what instructional technologies can be brought to address this search for particular contexts? While there is a wide range of instructional delivery technologies we can choose from, our choice must be carefully considered. The number of factors to be considered are too many to list here, but must include a cognizance of the nature of the content or skill that comprises the subject matter of instruction, the learners, the time and their place of study, and the costs of the delivery mode, both for the learners and the institution. This paper reports our experience of the first phase of a three-phased integration of ComputerMediated Communication (CMC) aimed at improving instructional effectiveness.

Interest in the application of some form of CMC in the enhancement of teaching-learning environments is currently widespread. This has led to the emergence of a growing body of literature on various aspects of CMC-based instructional delivery systems (for example, Mason and Kaye, 1989; Harasim, 1993; Mason, 1993; Wells, 1993). While there exists in this literature a great deal of information on the hardware and software requirements for 
such delivery systems, and numerous reports of applications in a wide variety of contexts, relatively little attention is focused on approaches to the integration of $\mathrm{CMC}$ in teachinglearning environments.

The project reported in this paper is about the design of instructionally effective CMCbased teaching-learning environments. The emphasis here is not on the hardware or the software requirements but on the organization and presentation of subject matter, the designed activities, and human factors which are often, in our experience, at the heart of the success or failure of such projects. A phased-integration of computer-mediated communications technology is described and also recommended to overcome an all-toopervasive mindset about teaching that is a carry-over from the conventional face-to-face classroom instructional situation.

\section{Teaching-learning orientation}

A considerable amount of research exists in favour of teaching-learning designs that engenders collaboration and interaction among the peer group (Ide et al, 1981). There is evidence, for example, that cooperative learning benefits learning for all except the most concrete, repetitive tasks with effect sizes as high as .80б (Johnson and Johnson, 1974). Allowing learners to exercise adaptive control over their learning process is also reported as having positive impacts on learning (Hannafin and Colamaio, 1988). CMC-based teaching and learning systems are characteristically designed to empower learners by allowing them a greater degree of adaptive control over their learning environment which is not feasible in conventional systems. This facility refers to the flexibility that learners may have in exercising as much control and autonomy over their learning, as and when necessary.

The hypothesized advantages of this electronic teaching-learning environment over the conventional system were several. For the learners it was intended to encourage them to move from:

- a defined learning space to an open, richer global resource base;

- an instructor controlled learning environment (that of a conventional classroom) to a collaborative and co-operative learning context; and

- being uncritical recipients of content to dynamic and participant explorers of the knowledge base.

The orientation of this project is derived from increasing evidence in favour of CMCbased collaborative learning and instructional environments. Its basic philosophy of flexible access and an instructor-learner negotiated teaching and learning process is at the heart of open learning systems, and increasingly now of conventional face-to-face teaching-learning environments which are also aiming for more flexible and adaptive formats (see the concept illustrated in Figure 1).

Figure 1 is a graphic representation of the teaching-learning environment that was designed and implemented as part of this study. Notice that the focus in the design is on integrating $\mathrm{CMC}$ in the teaching-learning environment as one of the resources available to the teacher and the learner. Students enter this instructional environment with variable 
Figure 1: Integrating CMC in the teachingleaming process

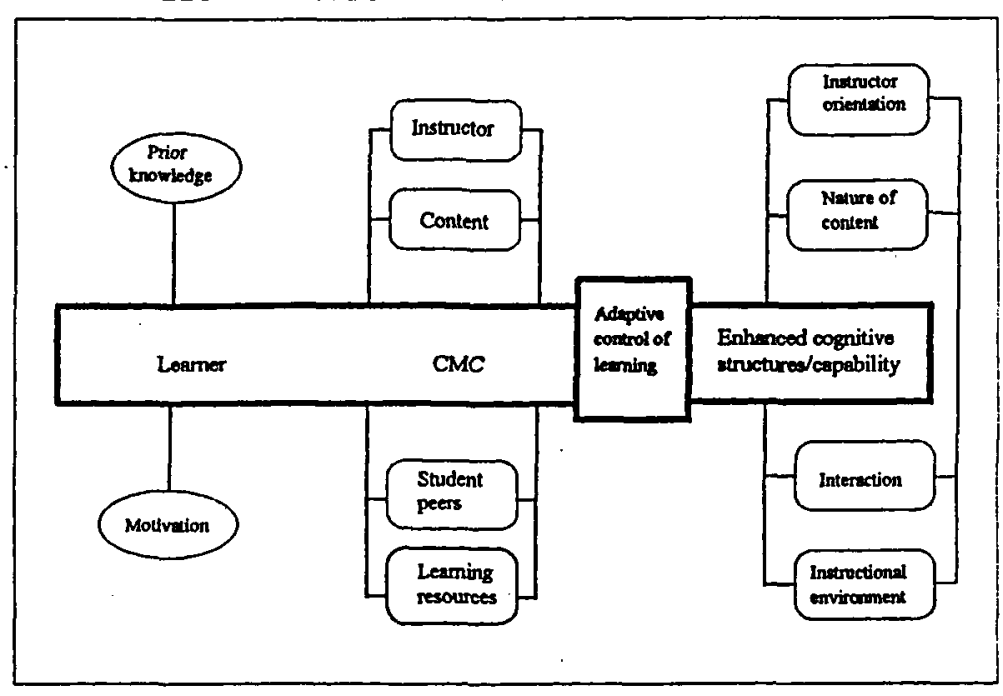

levels of prior knowledge in the use of $\mathrm{CMC}$ as a teaching and learning delivery medium, and also with variable levels of motivation to use it. Upon entry, they are confronted with an instructional environment that comprises the instructor, subject-matter content and CMC-based learning activities, conventional group lecture sessions, tutorial assistance, and other forms of individualized and group support. The goal of this instructional scenario is to allow the learner a level of flexibility that will enable adaptive control of the instructional environment. For the learner, this means seeking out and receiving as much instructional direction as is necessary, or assuming as much flexibility and freedom as one desires. Such autonomy should also allow the learners to use the instructional resources in a manner that will suit not only their place and pace of study, but also their learning styles and approaches to learning.

The intended outcomes of this instructional environment are several. These include foremost, for the learner, changed cognitive structures. This means a dramatically new and improved way of relating to content, storing it in memory and retrieving it. For the instructor, this arrangement implies new and improved ways of delivering content, and also improved ways of interacting with learners. The instructional delivery system also facilitates the arrangement of subject-matter content in new and interesting dimensions. Content can be organized in hypertext formats and include hypermedia to facilitate a richer and resourceful instructional and learning environment. All of these outcomes in the end lead to the creation of a new and improved instructional system that is more flexible, richer, and a realistic teaching and learning environment.

\section{Context and subjects}

The project reported in this paper was carried out in the context of a second-year unit in the Bachelor of Education program in the Faculty of Education at the University of Southern Queensland (Australia). The unit titled Teaching-Learning Studies focused on the classroom teacher and related issues like planning for teaching, instructional strategies, and classroom management. The project ran for a full 13 weeks in Semester 2, 1994. Subjects in the study were all the students enrolled in the unit. 


\section{Design}

In order to ensure systematic integration of $\mathrm{CMC}$ in the teaching and learning context, and to cope with the human factors pervasive in the situation, a three-phased model for implementing CMC was devised (see Figure 2).

\begin{tabular}{|c|c|c|c|}
\hline & $\begin{array}{c}A \\
\text { Document source }\end{array}$ & & $\begin{array}{c}\text { B } \\
\text { Communications }\end{array}$ \\
\hline 3 & Integrated MM & 3 & $\begin{array}{l}\text { Desktop video + } \\
\text { audiographics }\end{array}$ \\
\hline 2 & Hypertext documents & 2 & $\begin{array}{l}\text { Computer conference } \\
\text { (groupware) }\end{array}$ \\
\hline 1 & Mlerosoft Word & 1 & Microsoft Mall \\
\hline
\end{tabular}

Figure 2: The three-phosed CMC implementation

There are two components to each phase. The first component (A) concerns the organization and presentation of the subject matter. At the first level, all subject-matter content can be created, stored and retrieved as word-processed files. At the second level, the word-processed files are presented as hypertext documents to facilitate such uses as complete document search, a linked hypertext-type lesson structure, zooming-in and magnifying components, copy and paste to other documents, and insert 'post-it' notes and 'bookmarks', etc. The third level extends the hypertext document to include interactive multimedia and activate other programs such as a statistical package.

The second component of the system, the communications dimension (B), is similarly organized into three layers with the first level operating on electronic mail. The second level includes text-based conferencing systems and/or groupware, while the third level includes audiographics and/or desktop video conferencing.

This system provides for a 'stepwise' progression allowing advancement up the layer on one dimension while operating at a lower level in the other dimension (that is, hypertext documents and email - A2 B1), or integrated multimedia documents and conferencing A3 B2). The building of these steps is dependent on the technical infrastructure, skill level of students and the teachers, and more importantly, on the nature of subject-matter content and the desired interactions/activities between and among all participants.

Figure 3 represents the implementation model reported in this paper. The current project was designed to represent the first phase of this model. Core content in the model is held on a file server at level A1 as Microsoft Word documents. Level A2 uses CD-ROM as a storage device while level A3 utilizes greater mass storage devices such as Interleaf and Worldview. Communication at level 1 was via Microsoft Mail. At level 2, computer conferencing is envisaged, and at level 3 we are looking at audiographic communication. 


\begin{tabular}{|c|c|c|c|c|}
\hline & \multicolumn{2}{|r|}{$\stackrel{A}{\text { Document source }}$} & \multicolumn{2}{|r|}{$\begin{array}{c}\text { B } \\
\text { Communications }\end{array}$} \\
\hline & 3 & $\begin{array}{l}\text { I-CON Author + } \\
\text { Interleaf + Worddview }\end{array}$ & 3 & Smart 2000 \\
\hline & & Interleaf + Worldview & 2 & Computer conference \\
\hline $\begin{array}{r}\text { Figure 3: USQ } \\
\text { nplementation model }\end{array}$ & 1 & Microsoft Word & 1 & Mierosoft Mail \\
\hline
\end{tabular}

\section{Network configuration}

The phase 1 network configurations included 48633 EISA machines running Windows 3.0 with Microsoft Word 2.0 and Microsoft Mail. The core lecture content, including the learning activities for each week's study, was input as Word documents. All supporting reading materials were scanned and also included as Word documents. Students were required to $\log$ on, read each week's material, and then respond to the required learning activities via Microsoft Mail. Students in the project were registered on the local-area network individually, as well as under an alias which was the unit number. A user could therefore send a message to another directly by addressing a message to that individual, and also to the whole group by addressing messages to the alias.

\section{Learning activities}

Learning activities were generated by the instructor on a weekly basis. These activities were carefully designed to focus on the subject-matter content that was being covered during that week. In the first week, for example, students examined the topic of 'direct instruction' as an instructional strategy. Students were exposed to a discussion on the topic by the instructor. This was accessible as a Word document. After reading and synthesizing this material, students were required to respond to the learning activities that were generated by the instructor. Some activities required further reading, others required some field work, and others required summarizing or synthesizing.

\section{On-line response patterns}

Responses to these instructor-generated learning activities were transmitted via email to the student-group alias, which meant that it went out to all the students registered under that alias, including the instructor. Each student's response was therefore received by all the others in the group. Individuals could comment on all the responses that were made, and especially those that raised controversial or interesting issues. The instructor was in a position to observe the transactions that were going on via email and make his own contributions to the discussion. He would make his own contributions and also provide feedback to the student commentaries. Students could also send messages to individuals in the group, which would then comprise private communications as neither the 
instructor nor anyone else in the group could have access to that message, unless such a message was copied or forwarded to a third or fourth individual in the group.

\section{Data sources}

The objective of data collection was focused on the use and utility of the CMC-based delivery environment to the stakeholders (that is, instructors and learners). Hence mostly ethnographic data-gathering techniques were employed. These included in-depth interviewing of learners during and after the process, and also analysis of the texts from the online interactions. Questions asked, focused on various aspects of the delivery system and their interface with it. These included training in the use of email; the benefits and disadvantages of the delivery mode; the changed role of the instructor; the technologyhuman interface in online communication; impacts on learning outcomes; and problems with reading text online and other concerns relating to online communication. All interview protocols were tape-recorded and transcribed for further analysis.

\section{Analysis of Data}

The interviews were conducted using the following specific headings:

- training for the project;

- instructional delivery system;

- changed role of the instructor;

- technology-human interface;

- learning experiences.

\section{Observations}

\section{Preparatory training}

Learners reported being generally satisfied with the training that was provided in the use of email and online communication at the start of the project. There was a view that the training could have been more effective if it had been focused on specific needs of learners with variable levels of expertise with the email software and online communication. Most felt that a list (in print form) of the most common features of Microsoft Mail would have helped a lot. Some of the learners found certain components of the training redundant, and which could therefore have been eliminated with better design, delivery and coordination of the training programme.

\section{The instructional delivery system}

Participants felt that, while this new mode of delivery was 'different', it offered them much needed flexibility in the way of their place, pace and time of study. The general disposition was that this was a more time-efficient way of making content and instructor expertise more accessible to learners.

\section{Changed role of the instructor}

The use of $C M C$ in instructional delivery meant a significant change in the role of the human teacher. While CMC allowed the instructor a greater degree of interaction with 
individual students in the class, it also increased the instructor's workload. Even though learners realized that they were in contact with their instructor online 24 hours a day, the lack of face-to-face contact with the instructor in a $\mathrm{CMC}$-based instructional environment was missed by the students. There was an overall preference for retaining some face-toface contact in this predominantly electronic teaching-learning environment.

\section{Technology-human interface}

For the computer-literate, a CMC-based teaching-learning environment posed little problem. For the computer-illiterate however, this delivery mode was a source of much anxiety. The experience from this project revealed that several factors, if not carefully managed, could lead to the failure of such systems. These include issues relating to moderation of discussions, careful management of dominant personalities, and use of acceptable online conversation protocols. The asynchronous nature of the medium meant that a response from the intended recipients of a comment was not available or 'visible' immediately as is the case in face-to-face contexts. A few of the participants found it rather frustrating having to wait for 24 or more hours to get some response on their comments and queries.

Some students found reading and composing on-screen somewhat difficult. This was not unexpected. Initially, students preferred to read offline, compose their message on a word processor, then send the message as an attachment to an email message. This was a very time-consuming and tedious exercise that detracted from one of the obvious advantages of CMC systems, which is the spontaneity with which messages can be received and responded to. With some practice, however, students were able to read comfortably onscreen and also compose messages online.

\section{Learning experiences}

A majority of the students felt that one of the significant outcomes of the CMC-based delivery system was the shift in the degree of control learners could exercise in their learning. In the conventional face-to-face context, their instructors made a lot of the decisions about how and when things were supposed to happen. In the CMC-based system, the learners took over a greater degree of responsibility of their learning patterns, determining for themselves how and when things would be done. In some cases there was a commensurate increase in the amount of time students spent on studying. Students enjoyed the opportunity to interact with their peers in an on-going manner without being constrained by the hours of a lecture and tutorial session. Students reported getting more 'involved' in their study.

There were some negatives as well. One of the problems that seemed to concern students about online communication was that of being misunderstood and not able to defend or explain one's comments before the others were already in an attack mode. In a face-toface mode, if one felt that a comment was being misunderstood or misrepresented, there was the opportunity to correct it right there and then. In a CMC-based delivery mode, however, once a message had been posted, it was out in the open for its recipients to make whatever out of it, and it was only after some time, usually after a day or so, that one could correct oneself or clarify the misunderstanding of the others. An advantage of the possibility of this occurring was that learners were forced to think through their ideas and comments a lot more clearly before broadcasting these online. Some students found that in so doing they were improving their reading, writing and thinking skills. 


\section{Discussion}

A CMC-based delivery system is fundamentally different from a face-to-face teachinglearning environment. It carries with it, among other things, a uniquely different student and instructor mindset. Like any other instructional delivery system, a CMC-based teaching-learning environment has its strengths and weaknesses for learning and teaching outcomes. Its success, in these terms, is dependent on a number of design considerations that are unique to it. These considerations and their implications on teaching and learning in a CMC-based instructional delivery system are discussed in the remainder of this paper.

\section{Implications for system management}

An integral part of the success of a CMC-based instructional system is the function of the system manager. This is a person who is responsible for network upkeep and maintenance. Teaching and learning using CMC requires maintenance and support of email communications, word processing and transferring of files and material from wordprocessed documents to mail and vice versa. Many things do and can go wrong in this process, especially when dealing with students and instructors who lack much experience with operating in this mode.

It must be understood by all parties concerned that there is a protocol for online communication. These have been widely documented in the literature on CMC-based instructional systems (see Harasim, 1993). Stakeholders must be given initial training in these and their use with continuous support and reinforcement throughout the process. It must be understood that there will be a slow start, and that as students and instructors become familiar with the delivery medium, the pace will pick up. This underscores the need for that front-end training and on-going support to be carefully planned and executed.

\section{Implications for the instructor}

This refers to the perceptions that instructors hold about their roles as teachers. Usually these perceptions are a result of their own training as teachers, or requirements of their institution, and years of work experience in conventional or other educational systems. The principal tasks of teachers in conventional face-to-face instructional systems, for instance, are to prepare lectures, deliver them, hold small group tutorial sessions, and mark written assignments.

A CMC-based teaching-learning environment prescribes a different set of tasks for the instructor. These include, among other things, the development of the core content in advance of the actual instruction, presenting these online, and communicating with students on a continual basis in an asynchronous mode. The instructor takes on a facilitator's role, reading and responding to student's work and clarifying any misconceptions as they arise. The instructor is no longer tied down to lecture or tutorial times. All of this happens asynchronously and in a 'fluid' teaching-learning context. For some instructors this may mean additional work which may lead to the fear of being eternally 'on the job'. The truth is that a CMC-based instructional environment, if carefully planned and executed, has the potential to save time and effort for the instructor - time which previously had been taken up by routine and repetitive tasks such as grading paper-based work and answering generic types of queries. 
Many instructors also find this to be a rather 'loose' arrangement in which they relinquish their control over how students study. This is not always acceptable to many instructors who believe in retaining greater control of their classes. In the academic community, some of these perceptions are deeply ingrained and very resistant to change. Much of this resistance, especially towards new delivery technologies, can also be attributed to a lack of confidence on the part of instructors in their abilities, and a fear of having to learn a new skill. A CMC-based instructional delivery system poses this kind of threat to most instructors, especially those who have little or no proficiency in working with students online.

Our experience tells us that these are serious considerations that must be carefully planned for. Instructors have to be introduced to the radical shift in the mindset or orientation towards their teaching function in a CMC-based environment. Unless this shift in their mindset has taken place, success is far from certain.

\section{Implications for the learner}

Students, also, hold particular conceptions and a mindset about their own roles as learners and that of their instructors. Like instructors, they need to understand that a CMC-based teaching-learning environment shifts the bulk of the responsibility for learning onto themselves. This requires recognition of that responsibility on their part, and the assumption of an active role in the learning process. Students must understand that the instructor is no longer going to drive the learning for them, that they must learn to drive it for themselves.

\section{Importance of front-end training}

While an increasing number of the current generation of students are computer- and Internet-literate, there are still many among them who have no or only.negligible experience with computers and electronic networks. Experience derived from this project suggests that a carefully planned front-end training is imperative for both students and instructors involved in any CMC-based teaching-learning arrangement. Such a training program must be based on a thorough assessment of the needs of the participants. It is likely that these needs will be variable which would then necessitate individualized or small-group attention for particular or all aspects of the training. Yet while front-end training is a must, it is also certain that training up-front in the use of CMC will be insufficient. Training and continuing assistance will be necessary throughout the duration of such a project. Therefore, help with online instruction and the equipment will need to be available on a continual basis, either online or otherwise.

\section{Outcomes of CMC-based instruction}

A CMC-based instructional environment is fundamentally different from the conventional classroom-based system in very many ways. The difference between the two modes of instruction is most explicit in the manner in which content is delivered to the learner, the communication patterns between tutors and students and among the students themselves, the manner in which students access additional learning resources, and the manner in which assessment of learning is carried out. Most instructors who have been exposed to a CMC-based instructional system argue, although not initially, that the mode has had a significant and lasting impact on their overall approach to teaching and 
learning. Our experience suggests that this kind of an impact is manifested in several ways. Some of the more visible ones are discussed in the following sections of the paper.

\section{Expanded resource base}

Currently, the integration of CMC in instructional systems may include any one or more of a growing number of applications. These include access to electronic mail, bulletin boards, databases, networked CD-ROMs, network newsgroups, electronic discussion lists and computer conferences, File Transfer Protocol (FTP) facilities, and global search tools such as Gopher, Telnet, Knowboots, Netfind, Finger, and Archie. This list tends to be expanding all the time. With the help of an average personal computer and a communication connection, these applications have the potential of bringing to the learners an expanded learning-resource base which would be otherwise beyond their reach. The learning resources that these applications can bring to the learners include, inter alia, libraries and other sources of information at remote sites, access to international experts and research sites, other students, colleagues and peers, and access to relevant discussions that might be taking place in other parts of the world.

Our experience and those of others (Ferris and Roberts, 1994) show that access to an expanded learning resource base that is carefully selected can mean that learners are not only encountering more useful material than that specified in the curriculum, they are also covering the specified content and more material more rapidly than is possible in conventional systems. Learners shift from being passive receptacles to being active participants in the search for knowledge. Moreover, they learn how to acquire and use knowledge, and instructor-roles shift from dispensers of information to producers of environments which allow learners to learn as much as is possible on given topics.

\section{Co-operative learning environment}

Conventionally, co-operative learning refers to instructional and learning environments which are characterized by increased interactions among individual learners or among small groups of learners. This form of learning has its limitations no doubt, but its positive contributions to learning and instructional outcomes are reported to far outweigh its limitations or difficulties (Johnson and Johnson, 1984). A computer-mediated learning and teaching environment which depends upon interaction between databases, instructors, tutors and students, and also among students, is by definition a co-operative one. Computer-mediated co-operative learning and teaching has been shown to enhance learning outcomes in many different ways, including improvement in the quantity and quality of the learning experience (see Collis, in press).

Computer-supported co-operative instruction is also reported to have benefited the instructors. Ferris and Roberts (1994) report that as a result of the experience in the use of $\mathrm{CMC}$ in their instructional environments, instructors began to take a larger and more systems-oriented perspective of their contributions in the school, meeting each week in teams to plan an integrated effort. Problems that arose were dealt with by the teams rather than by the one instructor in the conventional system. Almost subconsciously, the roles of instructors in their classrooms got transformed into a more collaborative one.

\section{Authentic learning environment}

A computer-mediated learning and instructional environment is capable of reaching out to resources in remote locations, including people and sites, for relevant information that 
would otherwise be impossible to access by most students in the time that is usually available to them. As such, a CMC-based instructional system is capable of facilitating 'authentic' learning by enabling access to environments separated in time and place from one's home base. Realities can be created, in cyberspace in a manner of speaking, by electronic access to people in situ in different countries, cultures, systems and with access to libraries, databases and also discussions raging on the Internet.

\section{Authentic assessment environment}

Similarly, a CMC-based instructional environment is potentially capable of an assessment system that is authentic and dynamic in nature. Assessment can be authentic in the sense that it is situated and contextualized rather than contrived. It can be dynamic in that assessment is continuous and pegged to what students are doing in terms of their interactions and activities online. A variety of instructional strategies are open to instructors in CMC-based instructional systems such as setting up discussions, debates, tasks, project work etc. to which students are required to contribute. These contributions are asynchronous, and can be assessed by the instructor on an individual basis, including the provision of individualized as well as group feedback.

\section{Flexible learning}

Flexibility is a characteristic feature of a CMC-based learning and instructional environment. The integration of some form of $\mathrm{CMC}$ in teaching and/or learning means that the communication channels between the instructor and the students, and among the students, are not only open all the time, but asynchronous. With communication channels open, teachers and students have access to one another at any time of the day rather than waiting for the lecture, tutorial or consultation times to raise a query or share some interesting thought as these are occurring in the process of one's study. Messages, questions and contributions by students or teachers can be left on the network asynchronously, meaning at different times and from different locations. Open and asynchronous communications between the instructor and the students are the hallmarks of a flexible learning arrangement.

A CMC-based learning and instructional system is open also in terms of the many instructional strategies it can accommodate. These include individual searches of online databases, journals, libraries, and discussion groups. One-to-one communication may take the form of correspondence study, learning contracts, apprenticeships, and internships between the instructor-student, the student-expert, and also between students. One-to-many online communication may include lectures, symposiums, and panel discussions. Many-to-many techniques can include debates, simulations/games, role plays, case studies, discussions, project-based work, brainstorming, delphi and nominal group techniques, forums, and cognitive networking/mapping. This list of online activities is by no means exhaustive. It is an indication of what is possible in a CMC-based instructional environment with a little bit of imagination and creativity, mostly as part of instructional design and development.

Learning skills development

A CMC-based learning environment places the responsibility for learning more than ever in the hands of the learner. In so doing, the learner is greatly empowered, and also placed in a position of greater control of not only the amount but the quality of his or her 
learning. This autonomy allows learners (especially the enterprising ones) to explore, experiment, take risks and venture beyond that which is necessary. A natural outcome of this kind of initiative on the part of learners is enhanced learning skills relating to the search for and acquisition of knowledge. In conventional systems of instruction, much of this kind of autonomy is not possible due to a greater degree of instructor-control of learning, and also because of the lack of resources in the learners' immediate learning environment. With the help of CMC, that learning environment is now much larger and more accessible, allowing for a richer and larger resource base for the learners as well as the instructors.

\section{Concluding comment}

This project was implemented to ascertain, inter alia, factors that needed addressing when building a CMC-based learning and instructional delivery system. It was evident, from our experience, that in the initial stages students as well as instructors require an intensive training program to familiarize them with basic operations such as logging on and the use of passwords, moving between applications, such as from Word to Mail, and how to save and store material for future reference. Many students and instructors are still locked into the time and place concepts associated with lectures and tutorials. The issues of flexibility and openness must be carefully introduced to all stakeholders in such a project. Once familiar with this process of instruction and interaction, learners are able to work consistently at their own pace, and realize that instructors and tutors are interested in every individual learner's opinion and also in the collective views of the group. It is evident that a CMC-based instructional delivery system has the potential to facilitate that outcome as well as improve instructional effectiveness.

\section{Acknowledgement}

The authors are grateful to the Committee for Advancement of University Teaching (CAUT) for financial support in the conduct of this study. In addition, we thank John Elms and Ken Woolford (Faculty of Education, University of Southern Queensland) for their support.

\section{References}

Collis, B. (in press), 'Cooperative learning and CSCW: research perspectives for internetworked educational environments' in Lewis, R. (ed), Lessons from Learning, Amsterdam, North Holland.

Ferris, A. and Roberts, N. (1994), 'Teachers as technology leaders: five case studies', Educational Technology Review, 3, 11-18.

Hannafin, M.J. and Colamaio, M. (1988), 'The effects of variation of lesson control and practice on learning from interactive video', Educational Communication and Technology Journal, 35 (4), 203-12.

Harasim, L.M. (1993), Global Networks: Computers and International Communication, Cambridge MA, MIT Press. 
Ide, J.K., Parkeson, J.A., Haertel, D.D. and Walberg, H.J. (1981), 'Peer group influence on educational outcomes: a quantitative synthesis', Journal of Educational Psychology, 73 (4), 472-84.

Johnson, D.W. and Johnson, R.T. (1984), 'Cooperative small-group learning', Curriculum Report, 14, 1-6.

Johnson, D.W. and Johnson, R.T. (1974), 'Instructional goal structure: cooperative, competitive or individualistic', Review of Educational Research, 44 (2), 153-66.

Mason, R. (1993), Computer Conferencing. The Last Word, BC Canada, Beach Holme Publishers.

Mason, R. and Kaye, A. (1989) (eds), Mindweave: Communication, Computers and Distance Education, Oxford, Pergamon Press.

Wells, R (1993), Computer-mediated Communication for Distance Education: an International Review of Design, Teaching, and Institutional Issues, Research Monograph No. 6, Pennsylvania State University College of Education PA, ACSDE. 